\title{
Correlative Array Tomography - from 2D towards 3D
}

\author{
Alexandra F. Elli ${ }^{1}$, Robert Kirmse ${ }^{1}$, Jeff Caplan ${ }^{2}$, Cherish Warner ${ }^{3}$, Janine Sherrier ${ }^{3}$ and Kirk \\ Czymmek $^{4}$ \\ 1. Carl Zeiss Microscopy GmbH, Carl-Zeiss-Promenade 10, 07745 Jena, Germany \\ 2. 15 Innovation Way, Delaware Biotechnology Institute, University of Delaware, Newark DE \\ 19711, USA \\ 3. 15 Innovation Way, Delaware Biotechnology Institute, Department of Plant and Soil Sciences, \\ University of Delaware, Newark DE 19711, USA \\ 4. Carl Zeiss Microscopy, LLC, One Zeiss Drive, Thornwood, NY 10594, USA
}

Correlative microscopy combines information from light and electron microscopy (LM and EM) into one comprehensive dataset. The "Shuttle \& Find" interface from Zeiss was the first easy to use solution for imaging one and the same sample region in different microscopes, i.e. from widefield or confocal LSMs to environmental or field emission SEMs (FE-SEM). However, Shuttle \& Find is limited to 2D applications. Correlative Array Tomography (CAT) is a correlative volumetric microscopy method on a high-throughput basis. Ordered arrays of ultrathin, resin-embedded serial sections can be imaged with different microscopical modalities. Serial sections are prepared using an ultramicrotome and for correlative array tomography, sections were stained for imaging with a widefield microscope and a scanning electron microscope. CAT is a non-destructive method that allows multiple investigations of the same sample and even multiple successive fluorescent staining procedures [1]. Multiple staining cycles of a large number of antigens or the use of different fluorescent proteins followed by the ultrastructural investigation in a SEM enables the analysis of functional and structural information in the same context.

The software module “ ZEN Correlative Array Tomography” enables an easy and efficient workflow from LM (e.g. fluorescence) to FE-SEM or vice versa.

The first step after inserting the sample into one microscope (LM or SEM) is to perform a quick calibration to establish a coordinate system. Subsequently the software guides the user through an automated process to recognize the ribbons of serial section. Regions of interest (ROI) can then be selected in one section and these ROIs are then automatically duplicated to all other recognized section. Once this process is completed image acquisition of all ROIs is performed automatically by the software thus generating a 3D volume stack from the serial sections.

In the second step the sample along with the acquired data is transferred to next microscope (LM to SEM or vice versa). After the initial calibration of the coordinates the subsequent information on section and ROI position can be loaded and re-used for a fast set-up of the imaging parameters. Again, the microscope will then automatically acquire all images at the same regions that were already investigated. Finally a correlative 3D dataset can be created by first aligning both LM and SEM image stack separately. Afterwards the user correlates both volumes by identifying the same four reference points in both dataset thus obtaining a full $3 \mathrm{D}$ correlative volume. 
[1] K. D. Micheva and S. J. Smith, Neuron 55:25-36, 2007
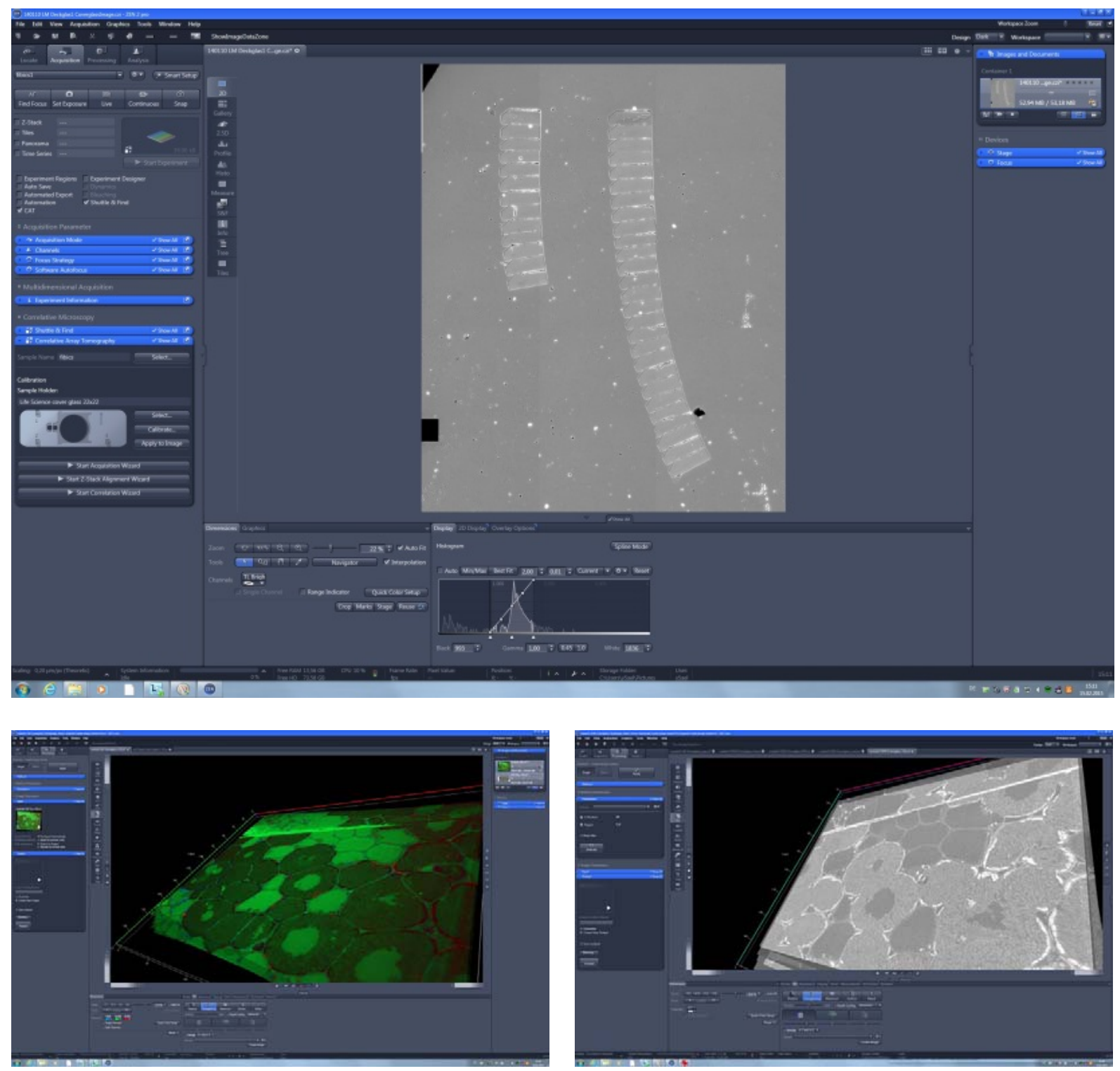

Figure 1: Overview image of individual ribbons on a glass slide detected with a light microscope. Reconstruction of 3D datasets of fluorescence images and images showing the ultrastructure of nodules. The purpose of the method is to reveal the precise localization of proteins in an ultrastructural context in 3D through correlated light and electron microscopy (CLEM) of zstacks. 